\title{
ESTUDIOS
}

\section{La misión de las universidades y escuelas de negocios de la Compañía de Jesús: retos y prioridades}

\section{Enrique López Viguria'}

Resumen: El objetivo del artículo es familiarizar a los lectores con la misión universitaria de la Compañía de Jesús, así como identificar sus principales retos y prioridades. También destacaremos la importancia del paradigma Ledesma-Kolvenbach como referencia, al abordar la misión de las universidades jesuitas y las escuelas de negocios y, en particular, el tipo de persona que la Compañía de Jesús aspira a educar. Para ello, revisamos textos de las últimas congregaciones generales (CG) así como los discursos clave de los últimos dos superiores generales de los jesuitas, cuyas enseñanzas son una "fuente de autoridad".

Palabras clave: Escuela de negocios, misión jesuita, paradigma Ledesma-Kolvenbach, universidad.

Fecha de recepción: 17 de noviembre de 2016.

Fecha de admisión: 8 de febrero de 2017.

The Jesuit University and Business School Mission: Challenges and Priorities

Abstract: The aim of the article is to familiarize readers with and further explore the Society of Jesus' (Jesuit) university mission, as well as identify its key challenges and priorities.
La mission des universités et des écoles de commerce jésuites: défis et priorités

Résumé: L'objectif de cet article est de familiariser les lecteurs à la mission universitaire de la Compagnie de Jésus (jésuites), ainsi qu'à identifier ses principaux défis et priorités.

' Secretario general de ESADE, Universidad Ramon Llull, Barcelona y UNIJES. Este artículo ha sido publicado también en Journal of Jesuit Business Education, 7/1, 2016, 1-21. 
We will also highlight the importance of the Ledesma-Kolvenbach paradigm, as a reference when addressing the mission of Jesuit universities and business schools and, in particular, the type of person the Society aspires to educate. For this, we review texts from the most recent General Congregations (GC) as well as the key speeches given by the society's last two Superior Generals; their teachings serving as a "source of authority".

Keywords: Jesuit mission, University, Business School, Ledesma-Kolvenbach Paradigm.
Nous mettrons en valeur également l'importance du paradigme Ledesma-Kolvenbach comme référence pour aborder la mission des universités jésuites et des écoles de commerce et, plus particulièrement, le genre de personne que la Société cherche à éduquer. Pour cela, nous travaillons sur les textes des dernières Congrégations Générales (CG) ainsi que sur les discours clés des deux derniers Supérieurs Généraux de la Société, en utilisant leurs enseignements comme «source d'autorité».

Mots clé: Écoles de commerce, mission jésuite, paradigme Ledesma-Kolvenbach, université.

\section{Introducción}

En este trabajo se persigue conocer y profundizar en la misión universitaria de la Compañía de Jesús, así como identificar sus principales retos y prioridades, destacando el paradigma Ledesma-Kolvenbach, una referencia obligada al tratar acerca de la misión de sus universidades y también de sus escuelas de negocios, acentuando la mirada en el tipo de persona que se quiere formar. Para ello se han revisado los textos de las últimas congregaciones generales de la Compañía de Jesús, así como los principales discursos y alocuciones de sus dos últimos superiores generales, ya que su magisterio es la siguiente "fuente de autoridad".

Vivimos en un mundo inmerso en un proceso de globalización sometido a profundos cambios y retos sociales, políticos, económicos, culturales, éticos y medioambientales. Un mundo que nos plantea nuevos dilemas y que nos brinda nuevas posibilidades de comunicación, de diálogo y de conocimiento. El impacto de la globalización alcanza y condiciona la vida de las personas, de las organizaciones y de las sociedades. En este nuevo mundo cualquier empresa u organización, máxime si tiene alcance internacional, ha de replantearse cómo llevar a cabo su proyecto, sus objetivos y medios de acción, en definitiva, el cumplimiento de su misión, de su razón de ser y existir.

El nuevo mundo plantea desafíos globales, y muchos de ellos han sido abordados en la agenda de Naciones Unidas (Nueva York, septiembre 2015) sobre los Objetivos de Desarrollo Sostenible. A todo ello se suman los problemas derivados de 
las crisis económicas y financieras, y los múltiples escándalos en el mundo de los negocios por asuntos de corrupción y de malas prácticas, es entendible que se haya puesto en cuestión el liderazgo, los valores y la responsabilidad de los directivos de muchas empresas y organizaciones, entidades financieras, instituciones públicas y grupos multinacionales.

Como efecto de todo lo anterior, es lógico que se haya propiciado una revisión del papel, la misión y el propósito de la educación superior y de las universidades, y muy especialmente de las escuelas de negocios, debatiéndose acerca de cuál ha de ser la misión de este tipo de instituciones para que su contribución sea socialmente responsable (PfefFer y Fong, 2004; KHURANA, 2007; StaRKEY y Tempest, 2008; Losada, Martell y Lozano, 2011; RAYMent Y SMith, 2013).

En este contexto, es pertinente dirigir la mirada hacia la Compañía de Jesús, una institución que cuenta con una de las mayores redes de universidades y escuelas de negocios del mundo, a través de la cual lleva a cabo su servicio a la sociedad y a la Iglesia en el campo de la ciencia y la cultura, de la docencia y la investigación.

El padre Adolfo Nicolás, superior general de la Compañía de Jesús (2008-2016), se hacía eco del debate mencionado anteriormente cuando invitaba a

los colegas de las aproximadamente 200 instituciones de educación superior, que funcionan bajo la bandera de la Compañía de Jesús [a] reflexionar juntos sobre cómo este nuevo contexto nos plantea el desafío de reorientar, en cierto sentido, la misión de la educación jesuita (NICOLÁs, 2010).

Para su predecesor, el padre Peter-Hans Kolvenbach (1983-2008), lo importante es responder a una pregunta que formula con cierta frecuencia, y que de forma genérica se puedes resumir así: ¿Qué tipo de hombres y mujeres necesitamos formar para que sean los líderes del tercer milenio? Para Kolvenbach, la razón de ser del compromiso de la Compañía en el ámbito universitario le lleva a realizar una afirmación que resulta esclarecedora en términos del sentido finalista de la misión universitaria jesuita:

El auténtico criterio para evaluar las universidades de la Compañía no es lo que nuestros estudiantes hagan, sino lo que acaben siendo y la responsabilidad cristiana adulta con la cual trabajen en el futuro a favor de sus prójimos y de su mundo (KOLVENBACH, 2008, 183).

Kolvenbach señala como un criterio decisivo a la hora de evaluar estas instituciones, no tanto lo que los estudiantes realicen en su periodo de formación y en su paso por los "campus", como lo que acaben siendo después de egresar de los mismos. 
La Compañía es una organización (orden) religiosa que, varios siglos antes de la aparición de la literatura de management, desde su misma fundación en el siglo $\mathrm{XVI}$, se revela como una organización fuertemente orientada a la misión (mission driven). Su compromiso misional también alcanza a la razón de ser y a la misión de sus universidades y escuelas de negocios, que viven las constricciones y condicionamientos del propio contexto donde operan y las del mundo globalizado, pero que han de llevar a cabo del mejor modo posible su propia misión en consonancia con la misión de la Compañía, la cual no les imparte directrices en un sentido normativo, sino más bien orientaciones e inspiración.

\section{La misión universitaria de la compañía}

Las universidades y los colegios aparecen como proyecto apostólico de la Compañía de Jesús años después de que Ignacio de Loyola la fundara. Ni en su etapa universitaria ni en la etapa inicial después de la fundación, Ignacio pensó y deliberó acerca de proyectos e instituciones educativas.

En palabras del anterior superior general, pasó un cierto tiempo desde su fundación para que

la Compañía reconociese la eficacia apostólica de la educación y crease sus propias instituciones de educación secundaria y universitaria donde se formasen sus propios escolares y alumnos seglares. De ese modo, la educación se convirtió en una prioridad apostólica (KolveNBACH, 2008, 36).

De hecho, la Compañía fue la primera orden religiosa en fundar una universidad al crear el Colegio Romano en 1551, matriz de la actual Universidad Gregoriana en Roma. Como apunta Kolvenbach, así

comienza una aventura que, a la muerte de lgnacio en mil quinientos cincuenta y seis, ha logrado ya la fundación de cuarenta instituciones de enseñanza superior, las cuales suman hoy más de doscientas dos universidades en todo el mundo (lbíd., 239-40).

Nicolás, en su alocución en México (2010) ante los representantes de la educación superior y de las universidades jesuitas del mundo, señaló que

es llamativo que en las Constituciones, Ignacio deja claro por qué se ha decidido por la idea de lo que él llama 'Universidades de la Compañía': La Compañía de Jesús acepta el 'cargo de universidades' para que los 'beneficios' de 'mejorar la enseñanza y la vida... se extiendan más universalmente'. El Bien más universal es lo que mueve a lgnacio a aceptar la responsabilidad de las universidades. (NICOLÁs, 2010, 10). 
La misión universitaria se enmarca en la misión intelectual de la Compañía de Jesús, en su apostolado o ministerio intelectual, que engloba tanto el ámbito universitario como el trabajo que desarrollan muchos jesuitas y muchas obras de la Compañía que no están encuadradas en el sistema universitario, como son las "casas de escritores", los centros e institutos de pensamiento y acción social, la extensa red de publicaciones y revistas, etc.

Kolvenbach en 2001 repasa la importancia concedida por las últimas Congregaciones generales al trabajo intelectual y universitario, y afirma que

no estará de más recordar que ya la CG 31 (1965) subrayó la importancia de este apostolado, insistió en la necesidad de preparar personal competente y pidió que se dieran facilidades a quienes trabajan en instituciones de la Compañía, o en otras universidades e instituciones científicas ajenas a la Compañía (KolvENBACH, 2008,197-8).

Más adelante prosigue diciendo:

La Congregación general 32 (1975), que para algunos pareció significar un cuestionamiento del apostolado universitario en aras del activismo social, en realidad insistió en el rigor científico de la investigación social, y en la necesidad de consagrarse al estudio austero y profundo requerido para la comprensión de los problemas contemporáneos. La Congregación general 33 (1983) volvió a recalcar la importancia del apostolado social y de la investigación, recomendando una mayor relación entre el campo intelectual, el pastoral y el social. La tensión y el malestar duraron muchos años, agravado por una desafección de los jóvenes con respecto a la educación. Esta situación, en general, parece hoy haberse revertido, aunque la disminución del reclutamiento jesuítico y la edad de los jesuitas en algunos países plantean un serio problema a mediano plazo (Ibíd., 198).

Cabe resaltar el hecho de que la Congregación general 34 (1995) dedique un apartado especial a "La Compañía y la vida universitaria" (decreto 17), en cuya introducción se recuerda que las anteriores Congregaciones generales habían tratado el tema de modo parcial y sin mayor profundización, aunque se destacara su importancia. Se subraya en el decreto la relevancia del trabajo universitario reconociendo que las universidades son encrucijadas de crucial importancia social, pero que el desarrollo que ha experimentado la educación superior de la Compañía de Jesús en los últimos años ha sido muy rápido, con un mayor tamaño y con mayor complejidad institucional y de estructuras de gobierno, dándose al mismo tiempo una disminución del número de jesuitas dedicados al mundo universitario, planteando todo ello un importante reto para la Compañía.

La CG 35 (2008), la última celebrada, considera el apostolado intelectual como una de las cinco preferencias apostólicas globales de la Compañía, resaltando que este apostolado ha sido una característica definitoria de la Compañía de Jesús desde sus comienzos y que 
teniendo en cuenta los complejos e interrelacionados retos que los jesuitas han de afrontar en todos los sectores apostólicos, la Congregación hace un llamamiento a reforzar y renovar este apostolado como un medio privilegiado para que la Compañía pueda responder adecuadamente a la importante contribución intelectual que nos pide la lglesia (CG 35, d.3, n.39, pp. 134-135).

En los principales discursos y conferencias de Kolvenbach y Nicolás acerca de la misión universitaria de la Compañía de Jesús se evidencia la importancia concedida a este ministerio desde sus orígenes, así como su relevancia cultural, social y apostólica en el presente. En las últimas décadas, ambos han expresado reiteradamente la importancia apostólica de la tarea universitaria y su estrecha relación con el núcleo misional de la Compañía de Jesús. Así, Kolvenbach señala: me atrevería a afirmar que la universidad jesuítica ha nacido 'ex corde Societatis' (del corazón de la Compañía) (KolveNBACH, 2008, p. 5). O Adolfo Nicolás, en su discurso en la celebración del $125^{\circ}$ aniversario de la Universidad de Deusto (Bilbao, 2011), que alude a la CG 35 y subraya la relevancia de la tarea universitaria para el conjunto de la Compañía de Jesús y de sus diversas obras:

Necesitamos el apoyo de la Universidad y su sabiduría, su capacidad de investigar y profundizar, su dominio de la técnica, etc. para sostener nuestras restantes obras, al servicio de la humanidad, especialmente de los pobres, y para afrontar los nuevos retos de la globalización y la economía (NICOLÁs, 2011, p. 6).

\section{Retos y desafíos misionales para las universidades y escuelas de negocios jesuitas}

A continuación, se ofrece una visión de cómo la Compañía plantea y afronta los principales retos y desafíos de su misión universitaria, que son una concreción de su misión universal en este ámbito específico. A este respecto, cabe preguntarse como lo hace Nicolás:

¿Cuáles son los retos de la Compañía? La única respuesta es: los retos del mundo. No hay otros retos. El reto es la búsqueda de sentido: ¿̇merece la pena vivir la vida? Y los retos de la pobreza, la muerte, el sufrimiento, la violencia y la guerra. Esos son nuestros retos. ¿¿Qué podemos hacer nosotros? (NICOLÁs, 2010, p. 10)

Estos retos y desafíos misionales también se plantean para todas sus universidades y para las escuelas de negocios jesuitas (en adelante, JBS). En su formulación puede apreciarse una clara invitación a la proactividad de las instituciones académicas jesuitas para encarar los retos con autenticidad y desde la perspectiva del "magis", tal como Nicolás señala: 
pidiendo que lo hagamos mejor o hagamos más de lo que ya estamos haciendo, o intentando hacer. Creo que es una manera válida de aceptar esos retos (lbíd., p. 13).

A partir de lo dispuesto en las últimas CCGG y en el magisterio universitario de Kolvenbach y Nicolás, se han identificado los siguientes retos y desafíos.

\section{I. Servir a la fe y promover la justicia}

Sin duda, la CG 32 marcó un hito para toda la Compañía al redefinir la misión desde el binomio "fe y justicia", afectando esta nueva orientación a todos los ministerios y campos de acción, constituyéndose en su eje integrador. Kolvenbach lo explica con claridad del siguiente modo:

La Congregación fue cayendo en la cuenta lentamente de que toda la Compañía de Jesús, en todos sus muchos ministerios, estaba siendo llevada por el Espíritu de Dios a tomar una orientación nueva. El fin principal de la Compañía de Jesús, el "servicio de la fe", debía incluir también "la promoción de la justicia". Esta nueva orientación no era sólo para aquellos que trabajaban ya con los pobres y marginados, en lo que se llamaba "el apostolado social". Más bien, este compromiso tenía que ser 'una preocupación de toda nuestra vida y constituir una dimensión de todas nuestras tareas apostólicas' (KOLVENBACH, 2008, p. 172).

La CG 34 prosigue en esta dirección postulando lo siguiente:

La Universidad jesuítica puede y debe descubrir en su propia contextura institucional y en sus genuinos objetivos un ruedo específico y adecuado para el encuentro con la fe que obra la justicia (CG 34, 1995 d. 17, n. 7, p. 347).

Las universidades jesuitas han de promover en sus objetivos y dinámica institucional un marco adecuado.

De este modo, sirviendo a la fe y promoviendo la justicia en línea propiamente universitaria, podrán descubrir nuevos horizontes y nuevos campos de investigación, enseñanza y extensión universitaria (lbíd., n. 10, pp. 348-349).

Servir a la fe y promover la justicia se convierte así en el núcleo misional de la Compañía de Jesús y también de su misión universitaria, y ello desde una opción preferencial por los más empobrecidos. Según afirma Kolvenbach, en la Universidad Iberoamericana, México, en 1990,

Una Universidad jesuítica hoy expresa su amor preferencial por los pobres (...) Esto, expresado en programas, a través de la cuidadosa selección de los proyectos de investigación, en las políticas institucionales, en los debates públicos y en los foros universitarios, constituye la sustancia de esta misión que tiene hoy en día la Universidad que queremos" (KOLVENBACH, 2008, p. 96). 
En este sentido, Nicolás se muestra consciente de que los retos que ello representa para la educación superior no son menores y de que cada universidad, atendiendo a su contexto y a sus posibilidades, ha de afrontarlos con determinación para proyectarse al porvenir. Así, afirma en 2013:

Al final, una universidad solo tiene futuro si ella sabe superar los desafíos que le aparecen, si revela su pertinencia para la sociedad de cada época y si consigue 'actualizar' su misión, contemporáneamente expresada en las orientaciones jesuíticas con el binomio del 'servicio de la fe y promoción de la justicia' (NICOLÁs, 2013, p. 9).

En 2014, se publicó un número especial de Promotio lustitiae titulado: "La Promoción de la Justicia en las Universidades de la Compañía", en el que se afirmaba que:

Los distintos sectores apostólicos cuentan con una variedad de capacidades necesarias para la promoción de la justicia. (...). Tal vez sean los sectores educativo (...) y universitario aquellos de los que más se puede esperar, siendo también los que disponen de una mayor cantidad de jesuitas y de recursos de la Compañía. (...) De ahí que la misión y la visión de una universidad deban incluir la promoción de la justicia, como expresión y servicio de la fe, modo de cuidar la creación, contenido del diálogo con otras religiones y motivación para la transformación de la cultura. (...) La tarea propiamente universitaria es un campo privilegiado para la promoción de la justicia en el largo plazo: su educación formativa tiene un importante influjo en lo que los estudiantes -hombres y mujeres- llegan a ser (Secretariado para la Justicia Social y la Ecología de la Compañía de Jesús, Promotio lustitiae, 2014/3, No. 116, p. 15).

Más adelante, en el mismo sentido, se alude al papel de la investigación y de la proyección social de la universidad jesuita en la promoción de la justicia. También cabe destacar la celebración de la Conferencia de la Educación Superior de la Compañía (Melbourne, julio, 2015), reunida bajo el título: Expanding the Jesuit Education Network. Collaborations for Social Justice, en la que se han presentado diversos proyectos e iniciativas en las que colaboran diversas universidades jesuitas de todo el mundo, así como el video corporativo "Social Justice and the Jesuit University" ${ }^{\prime 2}$.

\subsection{La identidad jesuita}

La evolución y el crecimiento de los centros de educación superior de la Compañía de Jesús en todo el mundo, en términos cuantitativos, de complejidad y de gobierno, planteó un desafío al que la CG 34 dio respuesta manifestando que hay que trabajar para mantener y aun fortalecer el carácter específico de cada

${ }^{2}$ Cf. http://www.luc.edu/melbourne/ 
una de nuestras instituciones: en cuanto jesuítica y en cuanto Universidad (CG 34, 1995 , d. 17, n. 5, p. 346), alertando de que en el futuro tanto el sustantivo como el adjetivo han de ser plenamente respetados.

El sustantivo garantiza el compromiso con la autonomía fundamental, la integridad y la sinceridad de una Universidad, precisamente en cuanto Universidad: un lugar de serena y abierta investigación y discusión de la verdad. Al mismo tiempo, indica los objetivos propios de toda Universidad (investigación, enseñanza, diversos servicios consecuentes a su misión cultual). (...) En cuanto jesuitas, buscamos el conocimiento por sí mismo, pero debemos interrogarnos de continuo sobre 'el para qué del conocimiento'. (...) que requiere de la Universidad armonía con las exigencias del servicio de la fe y promoción de la justicia establecidas por la CG 32, d.4. (CG 34, 1995, pp. 346-347).

Años más tarde, en una reunión internacional de la educación superior jesuita (Monte Cucco, Roma, 2001), Kolvenbach insiste en que en una universidad católica o de inspiración cristiana, bajo la responsabilidad de la Compañía de Jesús, no debe existir ninguna incompatibilidad entre las finalidades propias de la universidad y su inspiración cristiana e ignaciana, sabiendo mantener el equilibrio necesario entre la dimensión académica y la dimensión apostólica en sus actividades. Reconoce que

nunca como en estos últimos años las universidades de la Compañía han mostrado tanta preocupación por profundizar y poner de manifiesto su identidad católica, cristiana, jesuítica o ignaciana, según los casos (KolveNBACH, 2008, p. 196).

Y más adelante, en relación a la fidelidad a los ideales ignacianos que impregnan la misión de la Compañía de Jesús, afirma:

Los tiempos que nos ha tocado vivir son radicalmente distintos de los que vivió lgnacio de Loyola. Pero la 'ayuda de las almas', 'la mayor gloria de Dios y el bien universal siguen siendo el motivo fundamental del compromiso de la Compañía con la educación. El 'porqué' y el 'para qué' de nuestras universidades, el sentido profundo del trabajo que jesuitas y laicos cumplen en ellas, y la razón de la presencia de todos Uds. aquí, están anclados en esta visión de lgnacio (lbíd., p. 209).

Por su parte, Nicolás refuerza la importancia de la identidad y de la denominación jesuita, subrayando la necesidad de autenticidad en el quehacer educativo de la Compañía, al afirmar que:

La educación jesuita no es una etiqueta que dé entrada a determinados privilegios; es una referencia que no podemos utilizar con honestidad si no somos, en primer lugar y en toda circunstancia, personas de servicio, hombres y mujeres dispuestos a comprometerse gratuitamente (NICOLÁs, 2009b, p. 3). 


\subsection{El paradigma Ledesma - Kolvenbach}

Desde una concepción de la educación que ha de mirar a "toda la persona" hay que desarrollar una formación integral de la misma. La CG 34 alude a esta visión integral de la educación cuando postula

Una Universidad de la Compañía ha de distinguirse también por su oferta de formación humana, social, espiritual y moral, así como por la atención pastoral a sus alumnos y a los diversos grupos de personas que en ella trabajan o que con ella se relacionan (CG 34,1995, d. 17, n. 11, p. 349).

Para Kolvenbach:

Durante 450 años, la educación jesuita ha buscado educar a toda la persona, la persona completa, tanto intelectual y profesionalmente, como psicológica, moral y espiritualmente (KOLVENBACH, 2008, p. 182).

En este sentido holístico, las universidades y JBS han de distinguirse por orientar su quehacer docente e investigador desde una misión e identidad integrada por cuatro dimensiones fundamentales, que se integran y relacionan en el llamado paradigma Ledesma-Kolvenbach, una referencia obligada al tratar acerca de la misión universitaria de la Compañía. Se trata de la original reinterpretación realizada por Kolvenbach del para qué del compromiso de la Compañía de Jesús con la educación, a partir de la formulación de Diego de Ledesma, un teólogo y pedagogo jesuita del siglo XVI.

"Ledesma-Kolvenbach" es una expresión acuñada por el jesuita Melecio Agúndez, que en la introducción al libro Discursos Universitarios. P. Peter-Hans Kolvenbach, de cuya selección es responsable, revela lo siguiente:

hemos decidido designarle con este nombre compuesto porque, si bien la estructura cuatridimensional es original de Ledesma (siglo XVI), la traducción a lenguaje moderno y la terminología latina acuñada para Georgetown-Gregoriana (utilitas, iustitia, humanitas, fides) es producto made in Kolvenbach (citado en KolveNBACH, 2008, p. 24).

Kolvenbach en varios de sus discursos universitarios (Monte Cucco, Roma, 2001; IQS, Barcelona, 2006; Nôtre Dame de la Paix, Namur, 2006; y el dirigido al Consejo Directivo de Georgetown, Roma, 2007) hace referencia a cuál es el para qué o finalidad de la educación universitaria jesuita, reinterpretando la formulación de Diego de Ledesma. En este último discurso, que Agúndez califica como el "testamento universitario" de Kolvenbach, éste utiliza cuatro voces latinas para expresar las cuatro razones o motivos del para qué de la misión y el compromiso de la Compañía de Jesús en el ámbito universitario: utilitas, iustitia, humanitas y fides. A continuación, parafraseando a Kolvenbach sobre éste y otros discursos en que alude a ello, se sintetizan los aspectos principales de estas cuatro dimensiones: 
Utilitas: La finalidad práctica de la universidad, con la mira puesta en asegurar los conocimientos y competencias con los que sobresalir en el campo de especialización elegida, para prestar un servicio útil a la sociedad desde la competencia profesional.

lustitia: Educar mujeres y hombres en una solidaridad bien informada, para que promuevan todo lo que debe hacerse para construir unas justas estructuras sociales, económicas y políticas que defiendan nuestra humanidad común y una paciente promoción de la justicia.

Humanitas: La formación de personas más plenamente humanas desde el credo y tradición humanísticos de la educación jesuita, la libertad ilustrada de la conciencia, la libertad responsable de la palabra, el diálogo respetuoso y una profunda sensibilidad por todo lo humano.

Fides: La dimensión religiosa, en su más profundo significado, la entrega a la búsqueda de la plenitud de la verdad $y$, en este sentido, la universidad jesuita debe proponer la fe cristiana y ayudar a todo ser humano a encontrar a Dios.

Seguidamente, con la intención de mostrar sintéticamente y en paralelo la fórmula utilizada por Ledesma con la reinterpretación de Kolvenbach -utilizando textos de su discurso ante el consejo de gobierno de Georgetown, en 2008- se presenta la siguiente tabla:

\section{TABLA I. Una visión sintética del paradigma Ledesma-Kolvenbach}

\section{EL PARA QUÉ DE LA MISIÓN UNIVERSITARIA JESUITA}

Kolvenbach sobre Ledesma: "En un lenguaje bastante barroco, pero válido todavía hoy, ha puesto de relieve el alcance práctico y social, humanista y religioso de la educación ignaciana" (KOLVENBACH, 2008, p. 240).

\begin{tabular}{|c|c|c|}
\hline Paradigma L-K & Diego de Ledesma' (S. XVI) & Peter-Hans Kolvenbach ${ }^{2}$ (Ss. XX-XI) \\
\hline Utilitas & $\begin{array}{l}\text { Porque proveen a la gente } \\
\text { con muchas ventajas para } \\
\text { la vida práctica. }\end{array}$ & $\begin{array}{l}\text { La finalidad práctica de la universidad } \\
\text { jesuita, mediante una formación integral } \\
\text { y con sentido holístico. }\end{array}$ \\
\hline
\end{tabular}

\footnotetext{
${ }^{3}$ Citado en Kolvenbach, 2008, pp. 258-9.

${ }^{4}$ KolvenBACH, 2008, pp. 258-262.
} 


\begin{tabular}{|c|l|l|}
\hline lustitia & $\begin{array}{l}\text { Porque contribuyen al co- } \\
\text { rrecto gobierno de asuntos } \\
\text { públicos y a la apropiada } \\
\text { formulación de leyes. }\end{array}$ & $\begin{array}{l}\text { Educar mujeres y hombres para construir } \\
\text { unas justas estructuras sociales, econó- } \\
\text { micas y políticas que defiendan nuestra } \\
\text { humanidad común. }\end{array}$ \\
\hline Humanitas & $\begin{array}{l}\text { Porque dan decoro, esplen- } \\
\text { dor y perfección a nuestra } \\
\text { naturaleza racional. }\end{array}$ & $\begin{array}{l}\text { La formación de personas más plena- } \\
\text { mente humanas, desde el potencial de } \\
\text { la tradición humanística. }\end{array}$ \\
\hline Fides & $\begin{array}{l}\text { Y porque son la defensa de } \\
\text { la religión y nos guían con } \\
\text { gran seguridad y facilidad } \\
\text { en la consecución de nues- } \\
\text { tro fin último. }\end{array}$ & $\begin{array}{l}\text { La búsqueda de la plenitud de la verdad. } \\
\text { La piedra angular de la educación je- } \\
\text { suita es una Persona que enseñó, con su } \\
\text { palabra y su vida, la visión y los valores } \\
\text { de Dios, en orden a edificar y salvar a } \\
\text { la humanidad en todas las cosas. }\end{array}$ \\
\hline
\end{tabular}

Elaboración propia.

En este paradigma el para qué es único y sus cuatro dimensiones se comprenden en su conjunción. La utilitas invita a una profesionalidad competente que no se entiende sin una ciudadanía responsable, orientada por el sentido ético y de iustitia, siendo para ello clave forjar una humanitas personal plena, completa y consistente. Y las tres dimensiones interrelacionadas tienen su "desde dónde" en la fides, su principio inspirador y donde se imbrican para entenderse en profundidad, es decir, la apertura al sentido trascendente y a una vida guiada por el Espíritu que, en el caso jesuita, se concreta en la fe cristiana y en una espiritualidad específica, la que proviene de los Ejercicios Espirituales de San Ignacio.

Este paradigma orienta e inspira la misión de las universidades y JBS, no solamente en su acción educativa, sino también en las tareas de investigación, en las de proyección social y, en general, en su gestión institucional.

\subsection{Una aproximación del paradigma a las escuelas de negocios jesuitas}

El paradigma Ledesma-Kolvenbach ha sido mencionado y reinterpretado por Nicolás en varios de sus discursos. A continuación, centramos nuestra atención en el que pronunció en ESADE (Barcelona, 2008) por estar dirigido a una institución de referencia internacional en el ámbito de las escuelas de negocios.

En este discurso, Nicolás alude a las cuatro dimensiones del paradigma, núcleo fundamental del pensamiento universitario del padre Kolvenbach, y lo expresa 
mediante los cuatro "espíritus" que han de inspirar a cualquier JBS o universidad jesuita.

\section{Un "espíritu útil":}

La docencia realmente práctica debe orientarse a la formación de buenos profesionales que, siendo técnicamente competentes, sepan descubrir y vivir el sentido social de toda profesión: el servicio experto a la sociedad (...). El espíritu de la utilitas es un espíritu que engendra a profesionales conscientes de la diversidad de riquezas que puede producir el ejercicio de una profesión (...). Formar personas en la utilitas, formar personas "útiles", es quizás formar servidores. No formar a los mejores del mundo, sino formar a los mejores para el mundo. Con lo que la excelencia de un profesional se mide ante todo con el parámetro del mayor servicio a la familia humana (NICOLÁs, 2008, p. 176, resaltado por el autor).

Un "espíritu de justicia":

Como decía el P. Kolvenbach, los centros universitarios deben hacer posible que los estudiantes, 'a lo largo de su formación [...] dejen entrar en sus vidas la realidad perturbadora de este mundo, de tal manera que aprendan a sentirlo, a pensarlo críticamente, a responder a sus sufrimientos y a comprometerse con él de forma constructiva. Tendrían que aprender a percibir, pensar, juzgar, elegir y actuar a favor de los derechos de los demás, especialmente de los más desaventajados (cita de su discurso en Santa Clara, 2000) (lbíd., 177).

Al referirse al "espíritu humanista", Nicolás presenta una formulación que se ha convertido en una referencia educativa para la Compañía de Jesús:

En estos últimos tiempos, los jesuitas y laicos implicados en la educación universitaria hablan de cuatro características de la persona humana íntegra e integral, a partir de cuatro cualidades que empiezan por la letra " $C$ ". En efecto, el espíritu humanista genera personas conscientes, competentes, compasivas y comprometidas. Conscientes de sí mismas y del mundo en el que viven, con sus dramas, pero también con sus gozos y esperanzas. Competentes para afrontar los problemas técnicos, sociales y humanos a los que se enfrenta un profesional. Personas también movidas por una fuerte compasión. (...) la capacidad de sentir como propio el gozo y el dolor de los demás; la capacidad de ponerse en su piel; la capacidad de acompañarles y ayudarles desde dentro de la situación; la constatación de que el otro, cualquier otro, especialmente el otro que sufre, es mi hermano o mi hermana (lbíd. 178).

Un "espíritu de fe":

Los jesuitas hemos escuchado la Buena Noticia que Jesús anunció: Dios está cerca de todo hombre y de toda mujer. Y cuando las personas se abren a esta cercanía amorosa, salen de sí mismas y miran a los demás y al mundo 'de otra manera': como hermanos y hermanas de todos, como creación de Dios (...). La fe nos hace salir de nosotros mismos y nos ayuda a amar desinteresadamente (...) asumiendo pacientemente nuestros límites. 
$Y$ por esto la fe nos invita a superar los miedos que son inherentes a nuestra condición humana: el miedo, al dolor, a la enfermedad, a la inseguridad, a la pobreza, a la soledad (lbíd., 178-179).

Finalmente, Nicolás ofrece la clave de integración de las cuatro dimensiones o "espíritus" y lo hace desde la fides, afirmando que a la Compañía

este espíritu de fe nos impulsa a desarrollar paciente y apasionadamente "la utilidad, la justicia y la humanidad". La "utilidad" es también servicio a la creación continua del mundo. La "justicia" es acoger lo que Jesús llamaba "el Reino de Dios": la llamada a transformar el mundo en pos de la solidaridad y la reconciliación. La "humanidad" es creer profundamente en el amor de Dios al ser humano, y en su capacidad de trascendencia (lbíd., 179).

En la tabla 2 se sintetiza esta aproximación del paradigma Ledesma-Kolvenbach a las JBS.

\section{TABLA 2. Una aplicación del paradigma Ledesma-Kolvenbach a las escuelas de negocios de la Compañía de Jesús}

\section{EL PARA QUÉ DE LAS ESCUELAS DE NEGOCIOS JESUITAS}

Nicolás (2008) menciona y reinterpreta a Kolvenbach en varios de sus discursos, como el pronunciado en Barcelona, en ESADE, una escuela de negocios de referencia internacional.

\begin{tabular}{|c|l|}
\hline Paradigma L-K & \multicolumn{1}{c|}{ Adolfo Nicolás } \\
\hline Utilitas & $\begin{array}{l}\text { Un "espíritu útil": buenos profesionales, técnicamente competentes, al } \\
\text { servicio experto a la sociedad. Profesionales competentes y "útiles" } \\
\text { como servidores }\end{array}$ \\
\hline lustitia & $\begin{array}{l}\text { Un "espíritu de justicia": aprender a percibir, pensar, juzgar, elegir } \\
\text { y actuar a favor de los derechos de los demás, especialmente de los } \\
\text { más 'desaventajados'. Personas que sientan el sufrimiento del mundo } \\
\text { y lo piensen críticamente para comprometerse de forma constructiva. }\end{array}$ \\
\hline Humanitas & $\begin{array}{l}\text { Un "espíritu humanista", que genera personas conscientes, competen- } \\
\text { tes, compasivas y comprometidas" (La fórmula eufónica de las } 4 \text { C's) }\end{array}$ \\
\hline Fides & $\begin{array}{l}\text { Un "espíritu de fe". La Buena Noticia que Jesús anunció: Dios está } \\
\text { cerca de todo hombre y de toda mujer. Este espíritu anima a desarro- } \\
\text { llar paciente y apasionadamente la utilitas, la iustitia y la humanitas }\end{array}$ \\
\hline
\end{tabular}

No formar a los mejores del mundo, sino formar a los mejores para el mundo.

Elaboración propia. 


\section{Las nuevas "fronteras" para las universidades y las escuelas de negocios jesuitas}

La Congregación general 35 de la Compañía de Jesús tituló su decreto 3 de esta forma: "Desafíos para nuestra misión hoy. Enviados a las fronteras". "Las fronteras" es una expresión que hace fortuna y que según Nicolás es una de las expresiones "favoritas" de dicha CG y que

ha hecho diana en la imaginación de los jesuitas y socios en la misión. Una expresión a la que acuden muchos en la Iglesia y en otras congregaciones religiosas. Ciertamente no es propiedad exclusiva de la Compañía. Pero la expresión, en verdad, es muy evocativa (NICOLÁs, 2009a, p. 93).

En un mundo globalizado, con una fuerte influencia de las tecnología de la información y comunicación, Nicolás plantea los nuevos desafíos para la misión de las universidades jesuitas aludiendo a las fronteras de la profundidad y de la universalidad en sus discursos de Manila (2009) y México (2010), señalando que ambas son dos de las principales preocupaciones de la Compañía de Jesús.

\section{I. La frontera de la profundidad: saber para servir}

Para la Compañía, el apostolado intelectual es de capital importancia y constituye una de sus prioridades apostólicas como señala la CG 35, concediendo especial importancia a la investigación intelectual para conocer

las estructuras económicas, sociales y políticas en que se hallan inmersos nuestros contemporáneos" y reconoce que "la formación y la competencia profesionales deben estar ligadas con la legítima autonomía y la libertad responsable que son imprescindibles para progresar en la docencia y en la investigación (CG 34, 1995, d. 16, n. 3 y 4, pp. 334-5).

Como afirma Kolvenbach:

La calidad del servicio apostólico que preste la Compañía dependerá en gran medida de su rigor académico y del nivel de su investigación intelectual (KOLVENBACH, 2008, p. 198).

Y ello cobra una nueva dimensión en el mundo que se globaliza y que plantea nuevos desafíos para el conocimiento y la praxis, lo cual le lleva a recordar un criterio de discernimiento muy propio de la Compañía de Jesús:

En un mundo a la vez tan globalizado y diversificado, no hay que esperar que la Compañía dé normas universalmente válidas para todos los contextos. El criterio fundamental será siempre el del mayor servicio divino y bien de las almas (Ibíd., 199). 
Años después, en 2010, Nicolás resalta la importancia del trabajo intelectual en la universidad, señalando que la Iglesia ha reiterado en muchas ocasiones esta llamada a la Compañía de Jesús, refiriéndose a lo apuntado por Benedicto XVI en la CG 35:

El Santo Padre afirmó que la misión especial de la Compañía de Jesús en la Iglesia es 'en las fronteras', 'esos lugares geográficos y espirituales, donde otros no llegan o encuentran muy difícil llegar', e identifica particularmente como fronteras lugares donde 'la fe y el conocimiento humano, la fe y la ciencia moderna, la fe y la lucha por la justicia', se encuentran (NICOLÁs, 2010, p. 12).

En la CG 35 (2008) se confirma la misión de la Compañía de Jesús, pero teniendo en cuenta el nuevo contexto de la globalización, los grandes cambios económicos, tecnológicos y culturales, así como los nuevos desafíos y conflictos que afectan a la humanidad, esta Congregación realiza una llamada misional para establecer relaciones justas y de reconciliación "con Dios, de unos con otros y con la Creación", constatando que:

La tradición de los jesuitas de tender puentes superando las fronteras es algo crucial para el mundo de hoy (CG 35, d. 3, n. 17, p. 67), y urgiendo a los jesuitas y a quienes comparten la misma misión, en particular a las universidades y centros de investigación, a promover estudios y prácticas orientadas a enfrentar las causas de la pobreza y a mejorar el medio ambiente (CG 35, d. 3, n. 35, p. 75).

La frontera o desafío de la profundidad pone a prueba la misión universitaria jesuita. Desde una mirada profunda a la realidad del mundo, Nicolás plantea en el Ateneo de Manila, en 2009, el siguiente cuestionamiento a las universidades jesuitas, extensible a las JBS:

La presente crisis económica del mundo y el sufrimiento continuo de millones nos revelan, que muchas de nuestras antiguas soluciones no funcionan, y se necesitan nuevos intentos basados en nuevas y más creativas formas para entender las muchas y complejas realidades de la vida humana y el mundo: negocios, finanzas, cultura, la función del estado y la política, el medio ambiente, la familia, la migración, las relaciones internacionales y la cooperación de derechos y deberes humanos, y el verdadero significado de lo que es el ser humano. Aquí hay un claro llamamiento a la profundidad. ¿Cómo pueden las universidades, donde se encuentran tantos intelectuales tan dotados y tan altamente formados, nuestros maestros e investigadores, promover una reflexión e investigación aún más profundas en esas áreas tan cruciales de las que depende el futuro de un mundo mejor? (NICOLÁs, 2009a, p. 7). 


\subsection{La frontera de la universidad: ser red para servir}

Ya en 2001, ante el mundo global emergente, Kolvenbach afirmaba la necesidad de que la educación superior de la Compañía de Jesús le haga frente activamente, dando

respuestas creativas al radical cambio de época que estamos viviendo. Ignacio quedaría hoy fascinado ante el fenómeno de la globalización, con todas sus increibles oportunidades y sus terribles amenazas, y no rehuiría los desafíos que ella entraña. A las universidades corresponde un papel insustituible en el análisis crítico de la globalización, con sus connotaciones positivas y negativas, para orientar el pensamiento y la acción de la sociedad (KOLVENBACH, 2008, p. 201).

Refiriéndose también al fundador de la Compañía de Jesús, Kolvenbach señala que vivió él la tensión entre lo local y lo global, pensando a nivel global, pero actuando a nivel local (Ibíd., p. 203).

\section{La CG 35 puso de manifiesto que}

El nuevo contexto de la globalización requiere de nosotros actuar como un cuerpo universal con una misión universal, constatando, al mismo tiempo, la radical diversidad de nuestras situaciones. Buscamos servir a los demás en todo el mundo, como una comunidad de dimensiones mundiales $y$, simultáneamente, como una red de comunidades locales (CG35, 2008, d. 2, n. 20, p. 97).

La frontera o desafío de la universalidad también merece la atención de Nicolás en su discurso de Manila, cuando señalaba:

Creo que una frontera que estimula hoy la educación jesuita es la universalidad, en sentido ignaciano de amplitud de pertenencia y anchura de preocupaciones y responsabilidad (Nicolás, 2009a, p. 97),

apuntando que la preocupación de san Ignacio fue siempre el bien universal; quería que los jesuitas estuvieran siempre dispuestos a servir en cualquier sitio donde hubiera la posibilidad de la gloria de Dios. Más adelante, Nicolás recuerda que la CG 35 dio más auge aún a la universalidad ignaciana cuando subrayó la urgencia de una perspectiva universal que nos permita mirar más allá de nuestras estrechas preocupaciones para trabajar junto con otros. Reforzando la orientación de la CG 35, preguntaba:

¿Cómo pueden nuestras universidades jesuitas -el término "universidad" participa de la misma raíz que "universal"- responder a este llamamiento a la universalidad (lbíd., 102). 
Nicolás, desarrollaba también lo apuntado en la CG 35 en su discurso en México, en 2010, interpelando sobre los retos con que la globalización desafía a las universidades de la Compañía, tanto en su actividad propia como en su actuar conjunto, preguntaba $\dot{i}$ ué clase de universidades, con qué prioridades y directivas, llevaríamos, si estuviéramos refundando la Compañía de Jesús en el mundo actual? (NICOLÁs, 2010, p. 14) y afirmaba

quiero poner de manifiesto que el tema pide una respuesta común y universal, basada naturalmente en vuestras diversas perspectivas culturales, y teniendo en cuenta la Educación Superior jesuita en su totalidad, como sector apostólico. Nicolás plantea con rotundidad la decisiva importancia del trabajo en red: urjo a las universidades jesuitas a que trabajen para formar una red internacional operativa que se ocupe de temas importantes que conciernen a la fe, justicia y ecología, que son retos que trascienden países y continentes (lbíd., 13).

Finalmente, hay que reseñar el mensaje dirigido por Nicolás al Foro Mundial de la asociación internacional de JBS, reunido en Montevideo, en julio de 2015, cuyo tema era "Liderazgo e innovación para un mundo sostenible". Después de aludir a los desafíos que el papa Francisco presenta en su reciente encíclica, Laudato si' - Sobre el cuidado de la casa común, Nicolás afirma que

frente a estos importantes desafíos considero central el aporte y la reflexión que nuestras Universidades y Escuelas de Negocios hacen y pueden hacer en este asunto (Nicolás, 2015, 2)

y confía

que su trabajo siga dando fruto para hacer de nuestras Instituciones de Educación Superior mejores instrumentos al servicio de una 'casa común más cuidada', es decir de un desarrollo humano sostenible. (lbíd., 4).

Según Nicolás:

Necesitamos formar líderes que no solo sean excelentes economistas o administradores, sino que también tengan una visión a largo plazo del desarrollo y una mirada profunda sobre la realidad, que les permitan ver la íntima conexión que vincula toda la creación (lbíd., p. 1). Asegurar una formación que permita en nuestros docentes, investigadores y egresados un liderazgo de tal calidad humana y profesional que aporte realmente a un desarrollo auténticamente humano y sostenible. Sin convicciones arraigadas, nos quedamos en la superficie de las cosas; y sin profundidad terminamos siendo incapaces de ver más allá de los intereses inmediatos que hoy predominan en la mayoría de los grupos económicos. (...) que nuestros graduados tengan una visión suficientemente amplia y profunda de los problemas que atañen al desarrollo de los pueblos y adquieran las convicciones necesarias para ser capaces de resistir las presiones inmediatistas que hoy parecen gobernar las políticas y las decisiones económicas de distintas organizaciones y empresas (lbíd., 2). 
Finaliza su mensaje señalando que la 'globalización de la superficialidad' es un gran desafío para la educación de líderes que piensen con profundidad y se comprometan en las cuestiones que merecen realmente la pena para transformar y mejorar las sociedades.

\section{Conclusión: implicaciones para las escuelas de negocios jesuitas}

La Compañía de Jesús, y su vasta red de universidades y escuelas de negocios, es un "actor" mundial relevante en la educación superior. Su consistencia misional y su compromiso efectivo son incuestionables. En el campo de las JBS, su red de centros, personas y capacidad instalada es muy extensa, y su nivel de contribución social cuenta con notable reconocimiento público.

En el contexto del mundo global y ante los desafíos planteados a la misión universitaria jesuita, la Compañía de Jesús invita y urge a cada institución universitaria a realizar su misión en su propio contexto y circunstancias, llamando también a dar una respuesta común y universal desde una actuación conjunta como sector apostólico. Las JBS son un medio privilegiado para responder, desde la su propia tarea, a los problemas y desafíos que plantea el mundo actual.

En este artículo se han identificado una serie de retos y "fronteras" de la misión universitaria jesuita que, con la mirada puesta en las JBS, pueden resumirse así:

- Escuelas plenamente "universitarias", con lo que ello significa de autonomía y rigor en la búsqueda de la verdad, en la observación de estándares de calidad en su actividad docente e investigadora, y en su contribución y transferencia a la sociedad.

- También escuelas plenamente "jesuitas", es decir, orientadas por una misión marcada por el servicio a la fe y la promoción de la justicia. Con una visión holística de la educación para el liderazgo empresarial, que mira a la persona completa y al mundo como creación inacabada de Dios. Con una misión que "encarga" una tarea exigente de humanización: en las personas y profesionales que educa, en el saber que promueve y en el servicio a la comunidad humana.

- Una "tarea universitaria" concebida con profundidad, con universalidad y a través de un trabajo conjunto en red. Esta misión, el para qué de la Compañía 
en las escuelas de negocios, se expresa y alcanza la condición de referencia en el paradigma universitario Ledesma-Kolvenbach: formar para la utilitas, la iustitia, la humanitas y la fides.

A la vista de los retos y desafíos que se plantean, cabe preguntarse en este ámbito: ¿Cuál es el nivel de concordancia entre la misión universitaria jesuita y lo que se formula en las declaraciones de misión de las JBS? ¿̨Y entre lo que se conceptualiza y lo que se valora en la práctica? ¿2Qué papel juega realmente la misión en los procesos de planificación y evaluación estratégica de estas instituciones?

Dado el carácter "mission-driven" de las JBS ¿ Cómo se desarrollan los procesos de formulación, comunicación y socialización de su misión universitaria? ¿¿Qué medios e instrumentos la aseguran? ¿ Cuáles son los sistemas e indicadores para su evaluación? En este sentido, un ejercicio de interés consistiría en estudiar cómo se valora el desempeño efectivo de la misión de las JBS en los distintos niveles: órganos de gobierno y dirección, profesorado, "staff" y gestores profesionales, alumnos y participantes, los antiguos alumnos, el sector profesional, las empresas y los reclutadores, las instituciones públicas y las entidades sociales, etc.

La actuación de las escuelas de negocios en entornos muy competitivos, donde los rankings, las acreditaciones y la reputación juegan un importante papel, muy vinculado a la empleabilidad de los egresados ipuede explicar suficientemente la desatención de algunos aspectos misionales claves? O bien, ¿̇puede ser motivo de falta de alineamiento entre los órganos de gobierno y los directivos académicas de las JBS? Podría resultar de gran interés analizar los sistemas de evaluación, contribución y retribución de los equipos directivos de las JBS, con el fin de comprobar su alineamiento efectivo con la misión y con las prioridades estratégicas de sus instituciones.

Por otra parte, ¿ Cuál es el papel real y la operacionalización del paradigma Ledesma-Kolvenbach en su desarrollo y puesta en práctica en las JBS? ¿̇Con qué efectividad se asumen y desarrollan la iustitia y la fides, dos dimensiones claves de la misión jesuita? De hecho, la fides es una dimensión que no se traslada fácilmente a los modelos y perfiles de competencias que se evalúan en las escuelas de negocios y en las empresas. Es una prioridad disponer de modelos de competencias personales y profesionales que aborden específicamente elementos misionales de las JBS a partir de las dimensiones del paradigma Ledesma-Kolvenbach.

La red mundial de JBS tiene un extraordinario potencial. Los proyectos e iniciativas en marcha entre JBS y en el seno de la IAJBS son crecientes (Global Jesuit 
Case Series, Healing Earth, Jesuit Digital Network, Jesuit Commons, The Ignatian Spirituality Project, así como diversos proyectos y alianzas intercentros en ámbitos como social entrepreneurship, social justice, desarrollo sostenible y ecología, o de colaboración para impulsar JBS en África, etc.). La pregunta que late con fuerza en las JBS es: ¿¿Cómo seguir avanzando más y mejor para agregar y multiplicar el valor de las JBS al servicio de sus sociedades y de la humanidad en su conjunto?

En octubre de 2016 se reunirá la CG 36 de la Compañía de Jesús que, a buen seguro, ofrecerá una inspiración misional reforzada, nuevos caminos de liderazgo y renovados modos de proceder. Para las JBS puede ser una magnífica oportunidad para inspirar y renovar la "mirada" compartida y profundizar en su misión (o "para qué") en el desafiante mundo del siglo XXI.

\section{Referencias bibliográficas}

Compañía de Jesús (1995) Congregación General 34 de la Compañía de Jesús, Bilbao-Santander, Mensajero-Sal Terrae.

- (2008) Congregación General 35 de la Compañía de Jesús, Bilbao-Santander, Mensajero-Sal Terrae.

Khurana, R. (2007) From Higher Aims to Hired Hands: The Social Transformation of American Business Schools and the Unfulfilled Promise of Management as a Profession, Princeton (EE.UU.), Princeton University.

KolvenBaCH, P.-H. (2008) Discursos Universitarios. Selección e introducción de M. AgúNDEZ AgÚNDEZ, UNIJES-Provincia de España de la Compañía de Jesús.

Losada, C., Martell, J. y Lozano, J. M. (2011) "Responsible business education: Not a question of curriculum but a raison d'être for Business Schools", en M. MORSING y A. Sauquet Rovira, editores (2011) Business Schools and their Contribution to Society, 163-174,..., Sage.

NiCOLÁs PACHÓN, A. (2008) "Misión y Universidad. ¿̇Qué futuro queremos? Conferencia con motivo del 50 aniversario de ESADE", en ESADE, 50 años inspirando futuros. Barcelona, ESADE-Universitat Ramon Llull.

- (2009-a) "Alocución del P. General al Ateneo de Manila". Recuperado de http://pedagogiaignaciana.com/ 
- (2009-b) "Alocución del P. General al Congreso de la Unión Mundial de Antiguos Alumnos. Bujumbura (Burundi). Recuperado de http://www.Compañía de Jesús web.info/highedu/index.cfm

- (2010) "Profundidad, universalidad y ministerio académico. Desafíos a la educación superior jesuita de hoy. Encuentro Mundial de Rectores de Universidades Jesuitas. Universidad Iberoamericana de la Ciudad de México. Recuperado de http://www.Compañía de Jesús web.info/highedu/index.cfm

- (2011) Deusto. Recuperado de http://www.Compañía de Jesús web.info/ highEdu/doclist.cfm

- (2013) Recife, Brasil. Recuperado de http://pedagogiaignaciana.com/

- (2015) "Carta dirigida al rector de la Universidad Católica de Uruguay para ser leída en el Foro Mundial de la IAJBS" (Montevideo, 4-VII-2015).

Pfeffer, J. y Fong, C. (2004) "The business school business: Some lessons from the US experience": Journal of Management Studies, 41/8, 1501-1519.

RAYMENT, J. y SMITH, J. (2013) "The current and future role of business schools. Education + Training": Emerald Insight, 55/4-5, 478-494.

Secretariado para la Justicia Social y la Ecología de la Compañía de Jesús (2014) "La Promoción de la Justicia en las Universidades de la Compañía": Promotio lustitiae, n 116.

StaRkeY, K. y Tempest, S. (2008) "A clear sense of purpose? The evolving role of the business school": Journal of Management Development, 27/4, 379- 390. 